\title{
Paläoklimatologische Eindrücke aus Neuseeland
}

\author{
Von Martin Schwarzbach, Köln
}

\section{Mit 10 Abbildungen im Text}

$\mathrm{Z}$ us a mmenfassung. Einige Beobachtungen und Bemerkungen zum Klima und Paläoklima von Neuseeland aufgrund von Reisen und der Arbeiten von neuseeländischen Geologen werden mitgeteilt.

Einige Eigenheiten des Klimas. Wie Tasmanien hat Neuseeland relativ kühles und feuchtes Klima. Die Regenschattenwirkung der Gebirge ist besonders deutlich und damit im Zusammenhang der Wechsel der Vegetation. Im Vulkangebiet der Nordinsel gibt es Anfänge "edaphisch bedingter Wüsten“.

Die Gletscher an der Westk üste, besonders der Franz-Josef- und der FoxGletscher, sind eindrucksvolle Beispiele für das Nebeneinander üppiger, fast subtropischer Regenwälder (mit Baumfarnen) und Gletschereis und daher für den Paläoklimatologen und die Ausdeutung fossiler Gletscherspuren sehr wichtig. Beide Gletscher enden nicht weit vom Meer, fast $2000 \mathrm{~m}$ unter der Schneegrenze; sie haben sich in 21 Jahren um 1200 bzw. $1800 \mathrm{~m}$ zurückgezogen. Die Ursache für die tiefe Lage der Gletscherzunge liegt im hohen Niederschlag und in der Höhe und Steilheit des Gebirges.

Die Klimageschichte Neuseelands. Das Quartär wird nicht näher behandelt, der Einfluß junger tektonischer Bewegungen auf die Terrassen erwähnt. Das Klima des T e r tiärs war gemäßigt bis subtropisch und humid. Das Temperatur-Maximum lag nicht, wie in Europa und Nordamerika, im älteren, sondern (wie in Australien) im mittleren Tertiär. Das wird vom Autor zu erklären versucht durch die Kombination zweier Kurven: einmal der drift-bedingten Änderung der lokalen Breitenlage, zum anderen der allgemeinen Tendenz der Temperaturabnahme auf der Erde im Tertiär. $\mathrm{Da}$ mindestens Australien im Tertiär offenbar äquatorwärts wanderte, Europa dagegen allenfalls polwärts, verläuft die resultierende Kurve in beiden Gebieten verschieden. - Auch das Meso zoik um ist nicht tropisch und nicht arid. Das Perm ist möglicherweise etwas wärmer als in Australien gewesen.

Der Unters chied der Klimaentwicklung zu Australien ist auffällig. Australien wanderte seit dem Karbon-Perm von einer polaren Klimazone bis in die Subtropen und Tropen. Neuseeland dagegen verharrte möglicherweise \pm im gleichen Klimagebiet. Es läßt sich noch nicht sagen, ob der Unterschied nur vorgetäuscht ist (durch lückenhafte Überlieferung), oder aber dadurch bedingt wird, daß beide Gebiete unabhängig voneinander nach Norden wanderten (Australien schnell, Neuseeland langsamer).

$\mathrm{Sum}$ a ry. Some observations and remarks about the climate and paleoclimate of New Zealand, founded on journeys and the work of New Zealandic geologists.

Some peculiarities of the climate (fig. 1). New Zealand has a relatively cool and wet climate (similar to Tasmania at the present). There is a very conspicious difference between the very humid windward side and the arid lee-side of the Southern Alps (also in the vegetation, fig. 2). "Edaphically caused deserts" begin to develop in the volcanic area of the North Island (fig. 3).

The glaciers on the western coast of $\mathrm{New} \mathrm{Zealand}$ (fig. 4), especially Franz Josef and Fox Glaciers, are impressive examples for the coexistence of lush, nearly subtropical rainforests (with tree-ferns) with glacier ice (figs. 6,8 ). Therefore they are especially important for paleoclimatologists and for the interpretation of climatic indicators. Both glaciers have their tongues near the sea, nearly $2000 \mathrm{mts}$. below snow-line. Their recession (fig. 7) was 1200 and $1800 \mathrm{~m}$ respectively in 21 years. The cause for the low position of the tongues is to bee seen in high precipitation in connexion with the altitude and steepness of the mountains.

Climatic history of $\mathrm{New} Z \mathrm{Zealand}$. The Quaternary is not treated; it only is referred to the influence of recent tectonic movements on the terraces. - The climate of the T e r t i a r y was temperate to subtropical and humid. Maximal temperatures did not occur (as in Europe and North America) in the older, but (as in Australia) in the middle Tertiary (fig. 9). The author tries to explain this difference by the combination of 2 curves (fig. 10): one is the curve of changing latitude, caused by drift, the other is the general trend of the decline of temperature in Tertiary time. Because Australia obviously moved towards the equator, but Europe (if at all) towards the pole, the resulting curve is different in both continents. - Also the M e s o zoi c 
climate was neither tropical nor arid. Perhaps the Permian was a little warmer than in Australia.

Compared with Australia, the climatic history is distinctly different. Australia changed from a polar climate to a subtropical and tropical one since the Carboniferous-Permian period, but New Zealand seems to have remained more or less in the same climatic zone during this long time. We don't yet know whether the difference between New Zealand and Australia is only apparent (caused by gaps in our knowledge), or is caused by an independent northward drift of both regions (Australia quickly, New Zealand more slowly).

Den paläoklimatologischen Eindrücken von einer Australien-Reise 1963 (veröffentlicht im 3. Paläoklima-Heft der Geologischen Rundschau, Bd. 54, 1, 1965) folgt hier ein ähnlicher Bericht über eine zweite Reise, die 11/2 Jahre später, im Januar-Februar 1965, nach Neuseeland (und Tasmanien) führte. Einen kurzen Besuch hatte ich schon 1963 Neuseeland widmen können. Der Bericht ist ebenso zwanglos und unvollständig wie der erste. Wie in diesem werden - von den regionalen Beobachtungen ausgehend - einige Bemerkungen über allgemeinere paläoklimatologische Fragen angeschlossen.

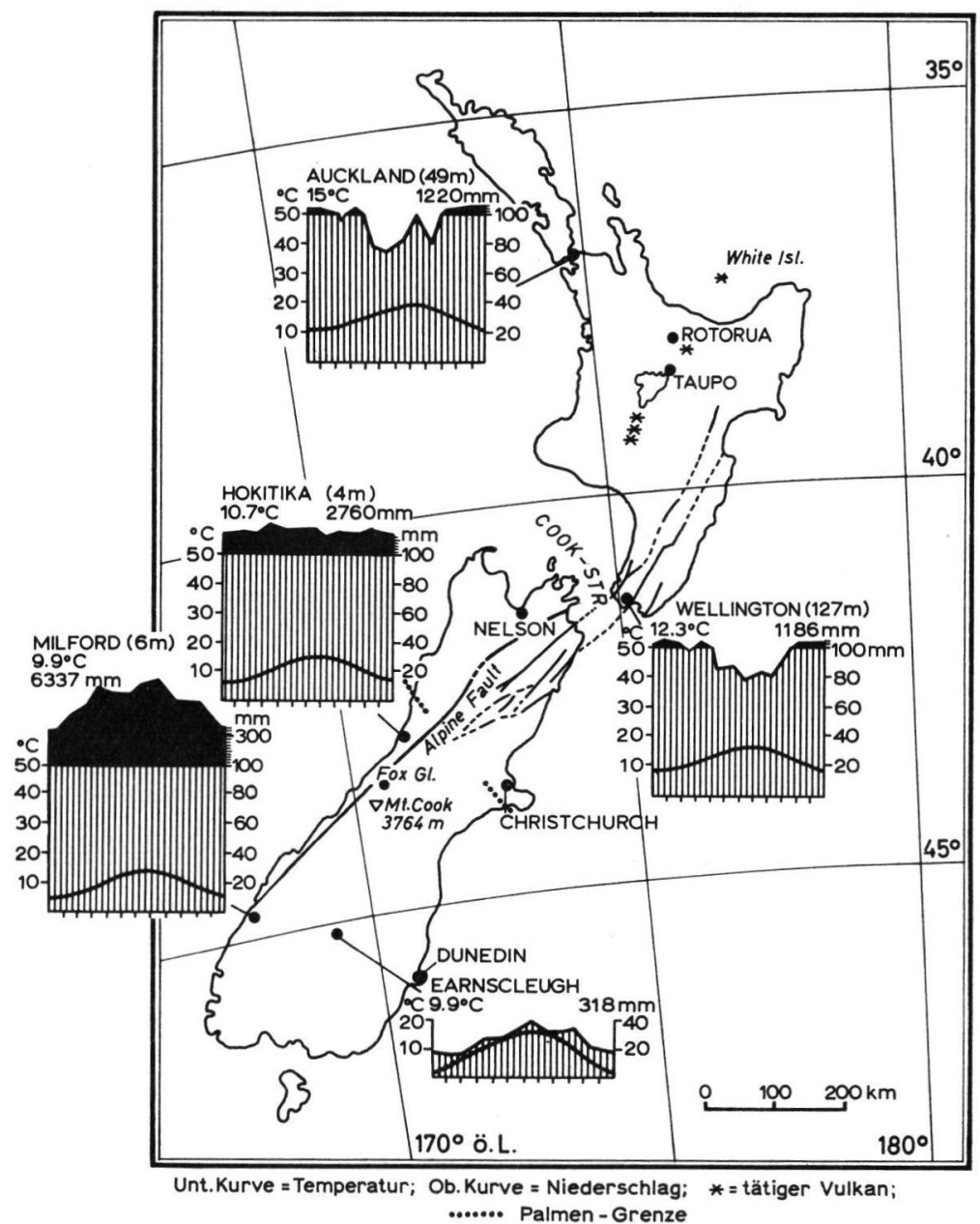

Abb. 1. Ubersichtskarte von Neuseeland mit einigen Klima-Diagrammen. Diagramme aus WALTER \& LiETH 1960. 


\section{Einige Eigenheiten des Klimas von Neuseeland}

Neuseeland hat ähnliche Breitenlage wie Tasmanien, ist aber noch weit niederschlagsreicher als dieser australische Staat. Wellington hat $12.9^{\circ}$ Jahrestemperatur (bei nur $8^{\circ}$ Unterschied zwischen den mittleren Monatstemperaturen von Januar und Juli), aber $1290 \mathrm{~mm}$ Niederschlag (zum Vergleich Hobart: $12.6^{\circ}, 580 \mathrm{~mm}$ ).

Bezüglich des $\mathrm{N}$ i ed e r s h la g s bestehen allerdings sehr große Unterschiede schon innerhalb Neuseelands. Besonders die Neuseeländischen Alpen, die die Südinsel in ihrer ganzen Länge durchziehen, sind angesichts der vorherrschenden Westwinde eine Regenscheide erster Ordnung. Milford Sound, im Regenluv der Westküste gelegen, hat die enorme Niederschlagsmenge von $>6000 \mathrm{~mm}$, aber in Teilen von Otago - östlich der hohen Berge - sinkt die Regenmenge auf nicht viel mehr als $300 \mathrm{~mm}$ herab. Damit steht Neuseeland den klassischen Beispielen für die Regenschat tenwirkung hoher Gebirge (z. B. Kauai in der hawaiischen Inselgruppe) nicht viel nach. (Abb. 1).

Das Pfla nzenkleid zeichnet diese Niederschlags-Unterschiede getreulich nach, und so ist eine Reise etwa von Hokitika an der Westküste über den Lewis-Paß nach Christchurch auch pflanzengeographisch höchst eindrucksvoll: dort üppige, subtropische Regenwälder mit Baumfarnen und Palmen (man vergleiche auch die Schilderung im nächsten Abschnitt und Abb. 8!), in höheren Lagen dichte Bergwälder mit der immergrünen Südbuche Nothofagus, und nach wenigen Stunden, östlich der Paßhöhe, eine im Sommer sonnenverbrannte, waldlose Steppenlandschaft mit zum Teil extremen Xerophyten (z. B. bei Rubus und der Robinie Carmichaelia) - so extrem, daß der deutsche Botaniker L. Diels fast bezweifelte (wie sein neuseeländischer Fachgenosse L. Cockayne hervorhebt), ob diese Anpassungen an ein Trockenklima wirklich dem heutigen Klima entsprächen (Abb. 2).

Außer dieser echten, klimatisch bedingten Aridität begegnet man - wie im Anschluß an frühere Ausführungen $(1963,1964)$ erwähnt sei - auch in Neuseeland derjenigen Trockenheit, die durch den durchlässigen vulkanischen Boden bedingt ist, also „e d a phisch bedingten Wüsten ". Die Autostraße Auckland-Wellington quert südlich von Tau po die östlichen Randgebiete des zeitweise aktiven Tongariro-Vulkans. Sie führt dort den bezeichnenden Namen „desert road“. Das ist zwar leicht übertrieben, denn von Wüste ist man auch hier weit entfernt, aber das Pflanzenkleid fängt tatsächlich

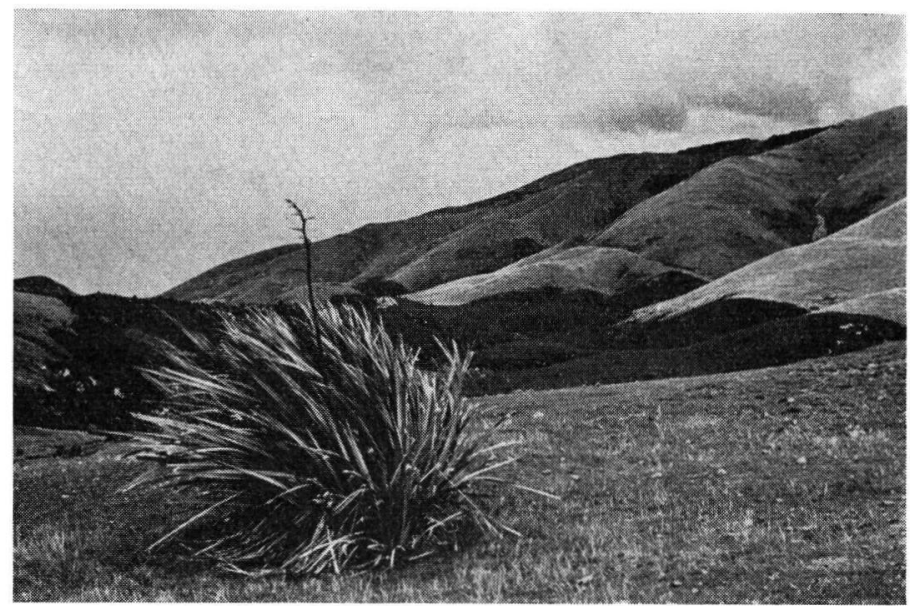

Abb. 2. Steppenlandschaft am Mt. Grey (nördliches Canterbury). Der Hang wird von einer rezenten Verwerfung gequert. Vorn Phormium (Neuseeld. Flachs). fot. 8-2-65. 


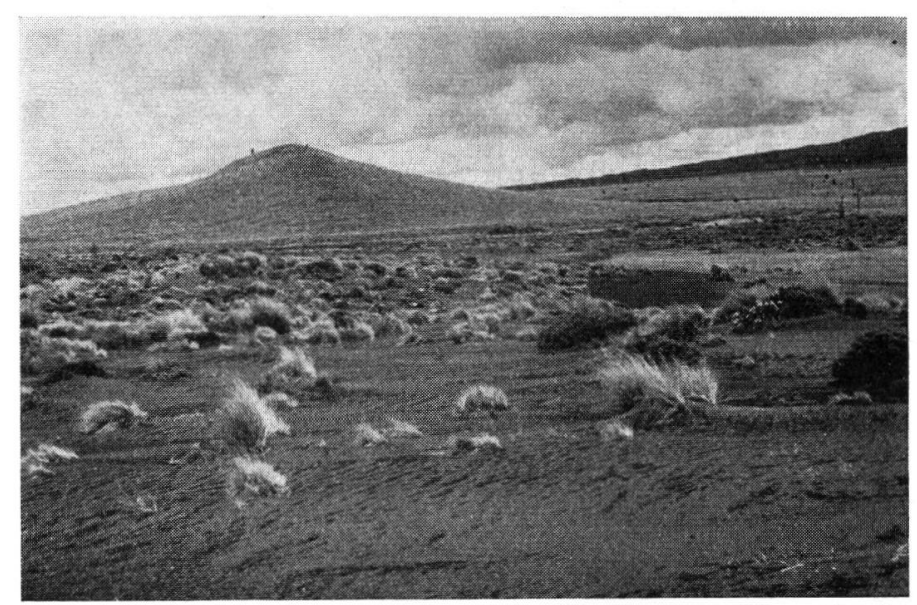

Abb. 3. Anfänge von edaphisch bedingter Wüste auf der Nordinsel von Neuseeland. Südlich Taupo (östlich vom Tongariro-Vulkan), an der „desert road“. fot. 28-1-65.

an, lückenhaft zu werden. Nackter, lockerer Boden zeigt sich überall zwischen den einzelnen Büschen von Gras, Aciphylla u. a. Der Untergrund wird hier vor allem von LaharAblagerungen gebildet (Abb. 3).

\section{Die Gletscher an der Westküste Neuseelands}

Die Neuseeländischen Alpen sind auch heute noch stark vergletschert (Abb. 4; neuere Angaben u. a. bei Kolb 1958, Odell 1960, Mercer 1962, Gunn 1964). Zu den eindrucksvollsten Vorkommen gehören ohne Zweifel Franz-Josef-und FoxG le t s che r an der Westküste der Südinsel. Zwar übertrifft der nach Osten abfließende Tasman-Gletscher beide erheblich an Länge, aber keiner der vielen Gletscher reicht so tief herab wie sie. Ihre Zungen enden in wenigen $100 \mathrm{~m}$ Meereshöhe und gelangen fast bis ans Meer - und das in einer Breite, die der von Florenz oder der Côte d'Azure entspricht!

So kommt es, daß sich üppiger Pflanzenwuchs und Gletschereis an der Westküste so unmittelbar begegnen wie sonst selten auf der Erde, und eine Wanderung von dem bescheidenen, nur aus wenigen Häusern bestehenden Touristen-Zentrum Fox Glacier auf den nahen, leicht zugänglichen Gletscher gleichen Namens bietet ein einzigartiges Erlebnis (Abb. 5-6; vgl. auch das Titelbild im Lehrbuch A. C. SEward's, zuletzt 1959!). In der niederschlagsreichen Küstenniederung gedeihen Regenwälder, die es an Üppigkeit mit tropischen Urwäldern durchaus aufnehmen können. Zwar sind wir hier schon jenseits der südlichen Palmen-Grenze (an der Küste bei Greymouth, $120 \mathrm{~km}$ weiter nördlich, trifft man noch zahlreiche NikauPalmen, Rhopalostylis sapida, die einzige Palmen-Art, die in Neuseeland heimisch ist). Um so üppiger treten nun die ähnlich malerischen hohen $\mathrm{B}$ a u $\mathrm{m} \mathrm{fa} \mathrm{r}$ e hervor, die zusammen mit der sonstigen artenreichen Flora ein undurchdringliches Dickicht bilden. Im Dämmer riesiger Bäume trieft es von Feuchtigkeit. Die dicken Stämme sind am Grund oft morsch und zerfressen. Vielfach stehen sie schon schräg, und zierliche Farnwedel hängen wie grüne Kulissen herab, oder sie liegen umgestürzt am Boden. Doch diese toten Stämme unterscheiden sich kaum von denen der lebenden Bäume: von Rinde sieht man in keinem Fall etwas, so dick und dicht ist der grüne Überzug von Flechten, Laub- und Lebermoosen, Farnen aller Art und oft auch von höheren Pflanzen. Von Ort zu Ort wechseln die Bäume, Sträucher und Epiphyten, für den Nichtbotaniker eine verwirrende Vielfalt. Die hohen, im Wuchs an unsere Eichen erinnernden Bäume, die sich da und dort über die andern erheben, erweisen sich durch die vereinzelten rotleuchtenden Blüten als Metrosideros, eine z. B. auch auf Hawaii häufige Myrtacee. 


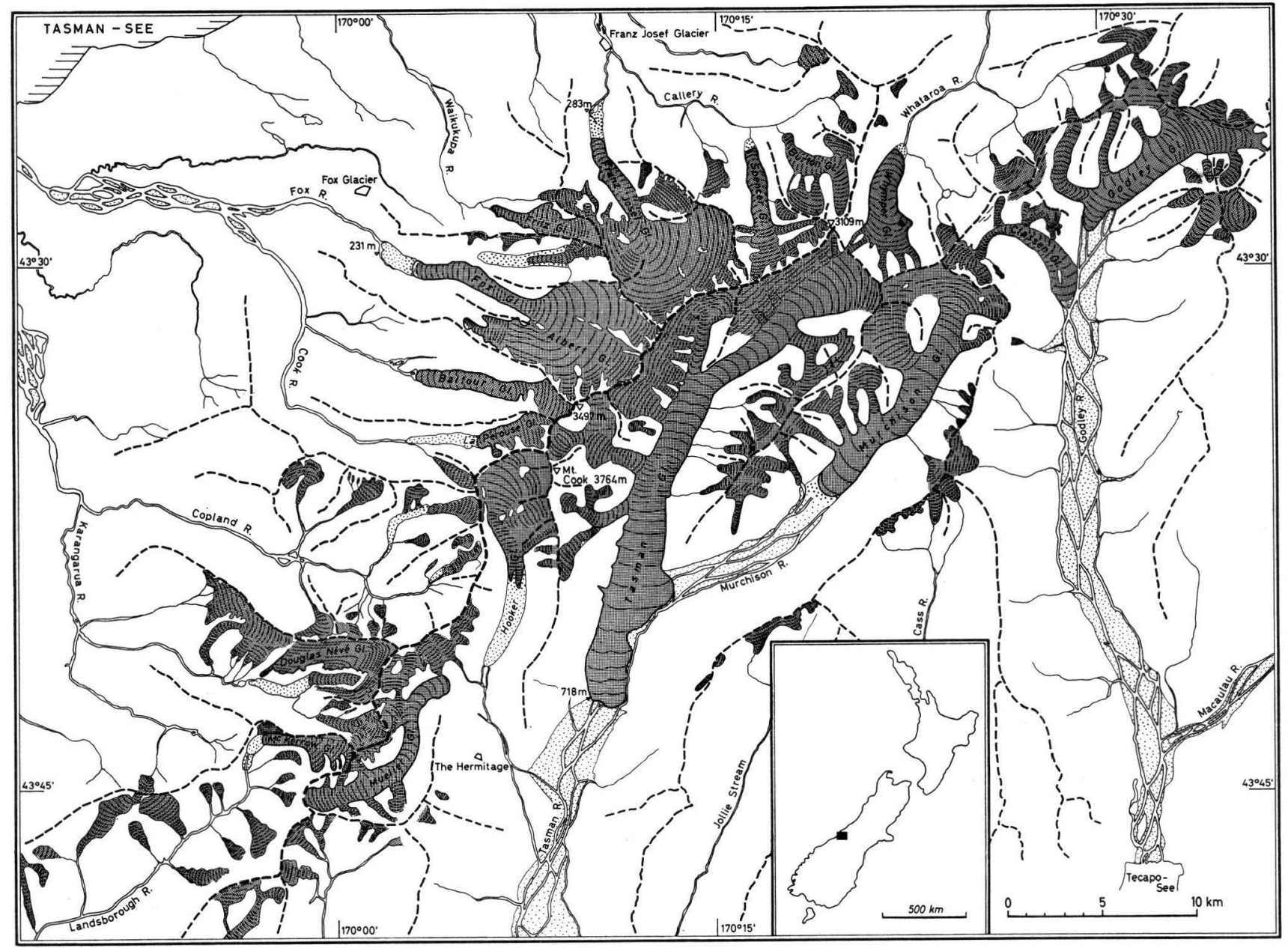

Abb. 4. Karte der heutigen Gletscher in den zentralen Neuseeländischen Alpen. Nach „Map of Westland \& Mt. Cook National Parks" (1:100 000). Zeichnung Dr. G. Schultz. 
Auch typisch südhemisphärische Nadelbäume treten stellenweise auf: mächtige Podocarpus, Dacrydium, dazu Pbyllocladus, bei dem anstelle der Nadeln fächerförmige Phyllocladien entwickelt sind (daher der volkstümliche, ganz treffende Name celery pine für Pbyllocladus).

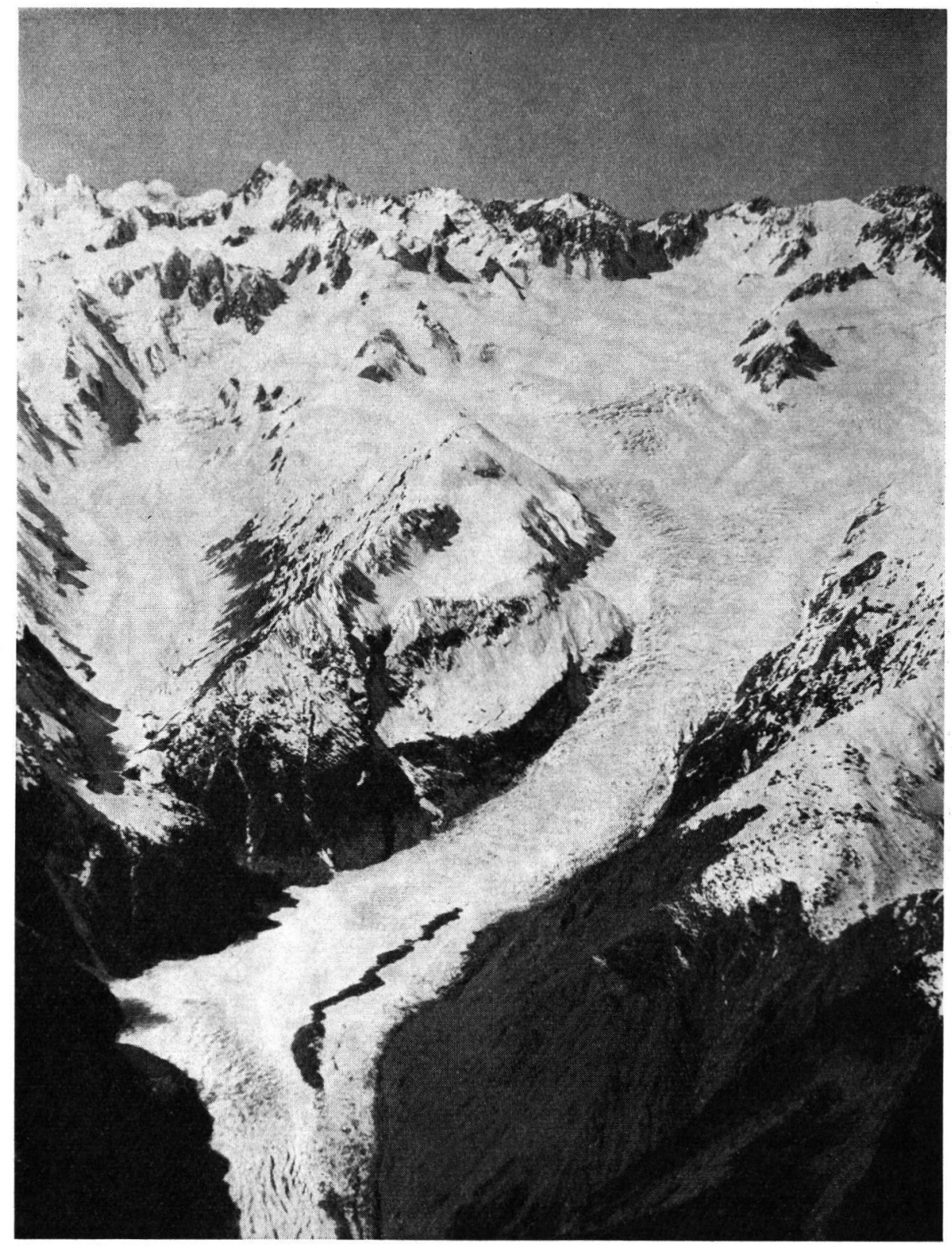

Abb. 5. Blick auf den Fox-Gletscher mit der Mt. Cook-Mt. Tasman-Kette. Vom Tourist Office, Wellington, freundlichst zur Verfügung gestellt. Klimatische Schneegrenze bei ca. $2100 \mathrm{~m}$; die Zunge reicht beinahe $2000 \mathrm{~m}$ tiefer. 
Diese grünen Urwälder treffen sich hier an der Westküste mit den weißen T a 1 glets chern, die von der Mt. Cook-Kette herabkommen. Im Gegensatz etwa zum Tasman-Gletscher sind sie schutt-arm. Der $11 \mathrm{~km}$ lange Franz-Josef-Gletscher endete noch 1956 in nur $230 \mathrm{~m}$ Meereshöhe (heute etwas höher). Ahnlich ist es beim Fox-Gletscher.

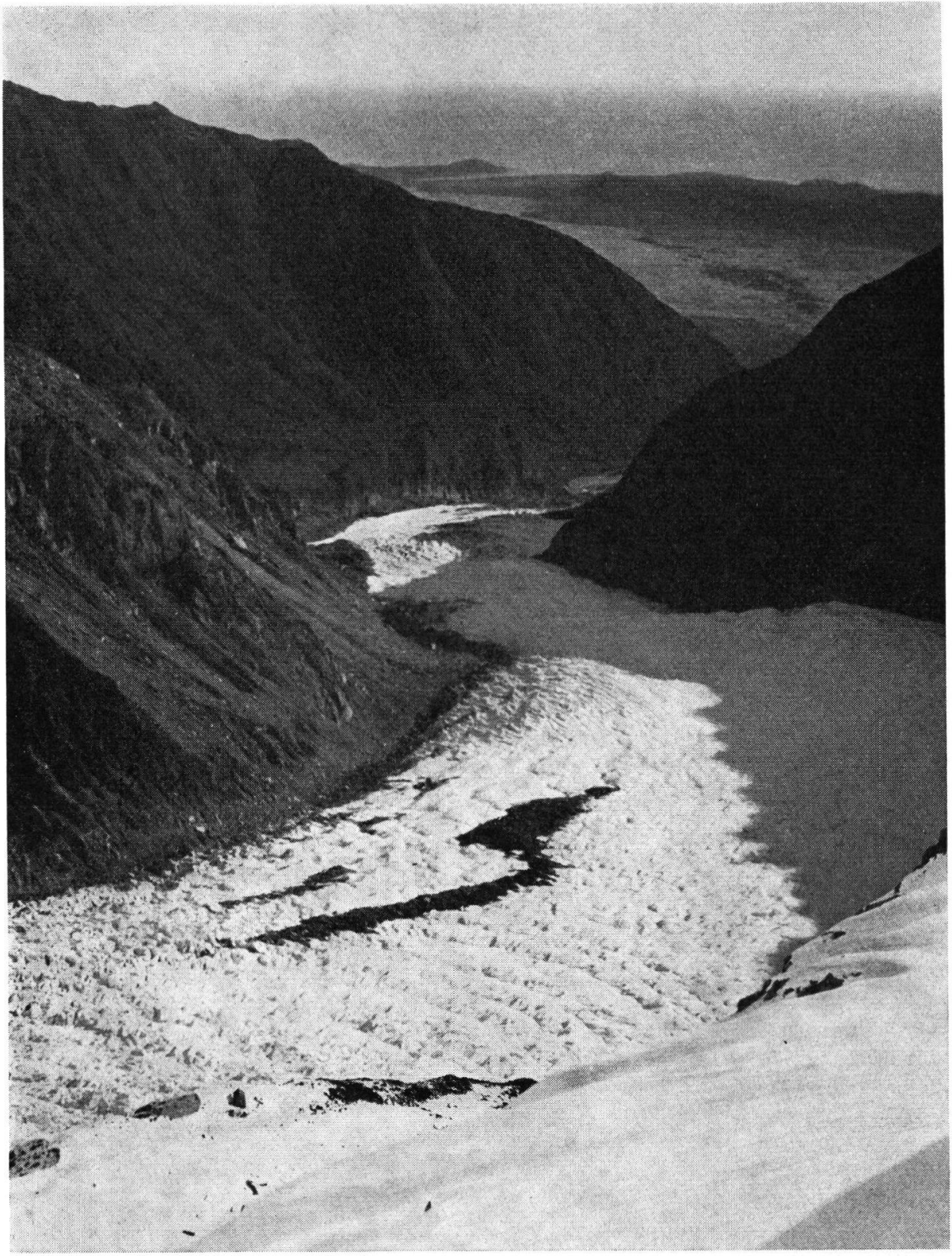

Abb. 6. Blick vom unteren Teil des Fox-Gletschers auf das Küstenvorland. Vom Tourist Office, Wellington, freundlichst zur Verfügung gestellt. Der Gletscher ist schuttarm. Im Zungen-Gebiet steilstehende kristalline Schiefer. Ein kahler Streifen am Hang-Fuß deutet auf den jungen Gletscher-Schwund; darüber dicht bewaldete Hänge. Im Hintergrund das Meer. 


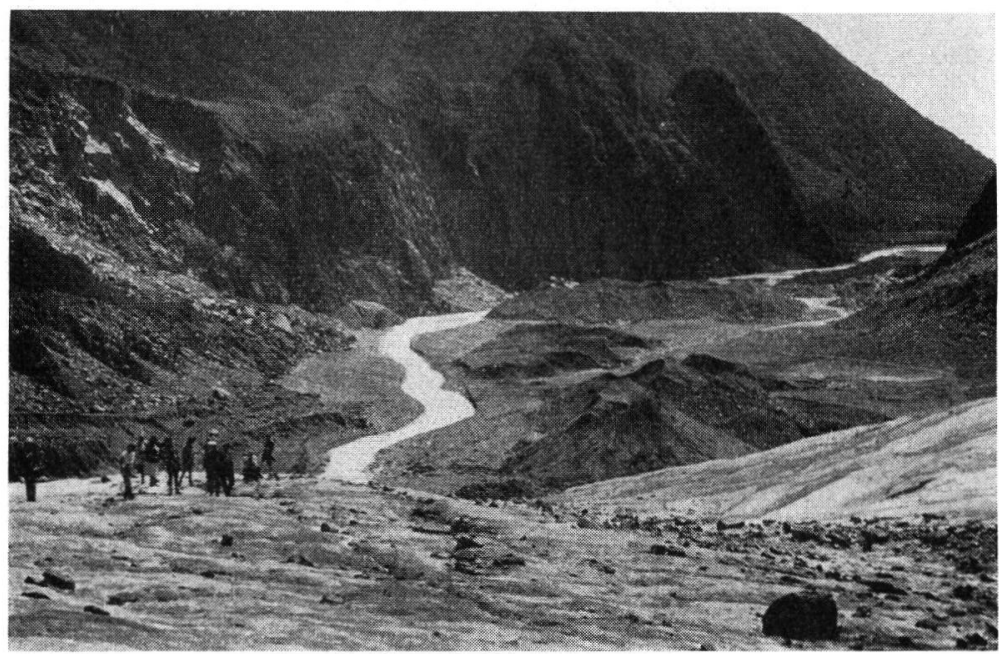

Abb. 7. Das junge Rückzugsgebiet des Fox-Gletschers, von der flachen Gletscherzunge aus gesehen. Frische Endmoränen mit Eiskern. fot. 5-2-65.

Seine Länge beträgt $15 \mathrm{~km}$; sein Firnfeld wird vom $3498 \mathrm{~m}$ hohen Mt. Tasman beherrscht. Die Schneegrenze liegt hier bei etwa $2100 \mathrm{~m}$.

Die schroffen Bergwände werden am Fox-Gletscher von steilstehenden kristallinen Schiefern gebildet. Waagerechte Gletscherschrammen zeugen von einem $\mathrm{fr}$ üh eren Gle t$\mathrm{sch}$ e r sta nd. Daß das Eis noch vor wenigen Jahren höher hinaufreichte, erkennt man auch daran, daß an den steilen Talwänden das grüne Pflanzenkleid erst in einigen $10 \mathrm{~m}$ über der flachen Gletscherzunge beginnt (Abb. 6), vor allem aber an den ungefügen Moränenhügeln, die bis über $1 \mathrm{~km}$ vor der heutigen Gletscherstirn im breiten, vom Fox River durchströmten Tal liegen (Abb. 7). Unter dem schwarzgrauen Schlamm und Schutt dieser Endmoränen kommt gelegentlich noch totes Eis zum Vorschein. Nach B. M. GUNN zog sich der Franz-Josef-Gletscher in den 21 Jahren zwischen 1935 und 1956 um 1200 m zurück, der Fox-Gletscher in diesem Zeitraum um $1800 \mathrm{~m}$ (davon $800 \mathrm{~m}$ zwischen 1956 und 1963!).

Unter den Kleinformen dieser glazialen Landschaft fallen neben den Gletschertischen und den Endmoränen noch flache, abflußlose Senken im Vorfeld des Gletscher auf. Die eine hat etwa $8 \mathrm{~m}$ Durchmesser und ist mit schlammigem Feinsand gefüllt, der allmählich eingesackt ist. Offenbar handelt es sich um ein T o t e is $10 \mathrm{ch}$.

Die beiden Westküsten-Gletscher sind für den Palä oklimatologen von b e s o n d e rer Wi chtigkeit. Sie beweisen, daß glaziale Erscheinungen und glaziale Sedimente unmittelbar zusammen mit Zeugen ganz und gar nicht-glazialen Klimas vorkommen können. Denn wenn auch die Baumfarn-Wälder von Fox Glacier keine Tropenhitze erfordern, so doch immerhin ein ganz normales gemäßigtes, beinahe schon subtropisches Klima. Das mahnt zur Vorsicht bei der Ausdeutung fossiler Klimazeugen. V e r steinerteBaumfarne schließen die unmittelbare Nachbarschaft von Gletschern nicht a us, und Tillite oder fossile Gletscherschrammen bekunden für ihren Fundort nicht unbedingt polares Klima. Ebenso bedeutungsvoll ist, daß hier Gletscher und Meer so nahe aneinanderliegen (beide Gletscher enden nur $15 \mathrm{~km}$ von der Küste entfernt in ganz geringer Meereshöhe). So etwas ist nicht überraschend in polaren Inlandeis-Gebieten, aber ungewöhnlich im gemäßigten Klimabereich. Wenn Tillite und marine Fossilien zusammen gefunden werden, bedeutet das also nicht in jedem Fall polares Inlandeis. Auch gewöhnliche Talgletscher können so etwas hervorrufen. 


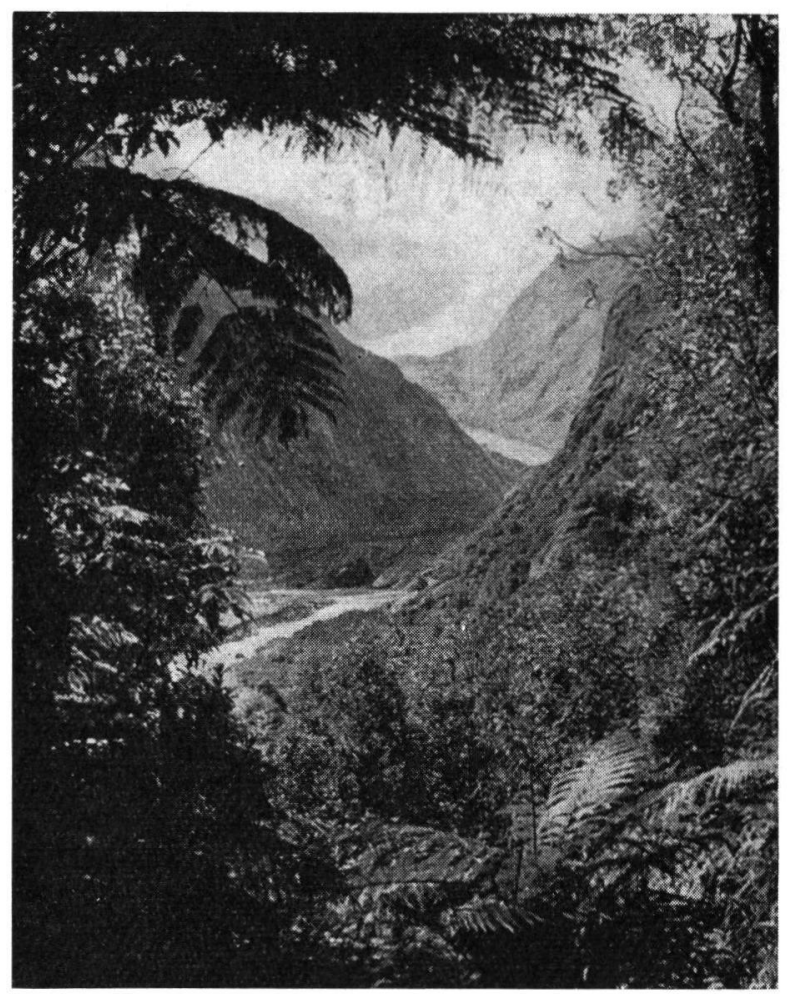

Abb. 8. Rückblick auf den Fox-Gletscher durch die Kulisse des Baumfarn-Regenwaldes. fot. 6-2-65.

Freilich bedarf es ganz besonders günstiger Bedingungen, damit das malerische Bild eines von Baumfarnen umrahmten Gletschers zustande kommt (Abb. 8). Neben ausreichendem Niederschlag muß ein hohes Gebirge vorhanden sein, das über die Schneegrenze aufragt. Nur dann kann sich Firn ansammeln, und nur bei steilem Relief kann ein Talgletscher weit unter die Schneegrenze in andere Klimabereiche und bis zum Meer vorstoßen. Außer in Neuseeland liegen dieser Verhältnisse - wenn auch offenbar nicht ganz so kraß - auch in Chile vor. Ch. DARwin hat das in seinem Reisetagebuch bereits lebendig geschildert.

Die hohe Lage der Schneegrenze in den Tropen schließt andererseits die Möglichkeit aus, daß wirklich tropische Klimazeugen und Moränen am gleichen Ort und zur gleichen Zeit fossil werden. Wenn also Korallenriffkilke und „Tillite“ zusammen angegeben werden, wird man immer an eine Fehldeutung denken müssen, d.h. an „Pseudotillite“.

\section{Die Klimageschichte Neuseelands}

Qu a r tär

Über das Quartär soll hier nichts Näheres gesagt werden. Darüber liegen ausgezeichnete, gründliche Untersuchungen neuseeländischer Geologen vor (so von C. A. FLEMING, Maxwell Gage, J. D. Raeside, R. P. Suggate, R. W. Willett und anderen), und P. Woldstedt hat in seinem Buch kürzlich einen Überblick gegeben. In besonderen Arbeiten (1962) wies er vor allem darauf hin, daß der quartäre Klima-Ablauf in Neuseeland (und Australien) offenbar ganz dem in Europa entspricht. Für das Postglazial ist das 
durch pollenanalytische Untersuchungen schon seit 1936 (L. CRanwell \& v. Post) bekannt. Die engen Beziehungen zwischen junger Klimageschichte (sowie anderen jungen geologischen Erscheinungen, besonders des Vulkanismus und der Tektonik) und der heutigen Oberflächengestalt haben durch C. A. Cotron eine hervorragende Darstellung gefunden; sein Lehrbuch der Geomorphologie (7. Aufl. 1958) erläutert beinahe alles, womit sich Geomorphologen beschäftigen, mit instruktiven Bildern aus Neuseeland.

T e r r a s sen sind häufig entwickelt, manchmal ungewöhnlich schön. An ihrer Entstehung dürften tektonische Bewegungen vielfach wesentlich beteiligt sein. Ebenso sind Flußterrassen nicht selten von ganz jungen Verwerfungen durchsetzt. Neuseeland gehört zu den wenigen Gebieten der Erde, wo Erdbeben noch heute echte Verwerfungen an der Erdoberfläche hervorrufen. Wellmann, Lensen u. a. haben Beispiele dafür beschrieben; auf das lebendig geschriebene Buch des neuseeländischen Seismologen G. A. EIBY (1957) sei ebenfalls hingewiesen. Die jungen Bewegungen dürften gelegentlich die Eingliederung mariner Terrassen erschweren.

\section{Tertiär}

Auch die Klimageschichte des Te r tiärs läßt sich gut rekonstruieren. Nach C. A. FLEMING und anderen kann man für die einzelnen Stufen annehmen:

Paleozän-Eozän: Meerestemperaturen nicht höher als $10-15^{\circ}$; erst gegen Ende wird es wärmer.

O l i g o z ä n : Keine Nummuliten und Riffkorallen, d. h. keine tropischen Bedingungen. Fossile Pinguine und Wale sprechen eher für kühlere Einflüsse. Auf dem Land deuten Pollen der heute in Neuguinea und Neukaledonien vorkommenden Nothofagus brassiGruppe (und die Seltenheit von Nothofagus fusca u. Coniferen) nach Fleming auf ein Savannen-Klima, „trockener als in fast allen übrigen Perioden der Klimageschichte Neuseelands."

Unter-Miozän: Die Nordinsel mit subtropischer mariner Fauna (mit indopazifischen Elementen), Höhepunkt der mitteltertiären Wärmezeit. Nothofagus fusca, Podocarpus, Palmen, Farne werden auf der Nordinsel häufiger, offenbar infolge erhöhten Niederschlages (der seinerseits durch verstärktes Relief verursacht wurde); doch wird diese Tendenz gegen Ende wieder rückläufig.

In diese Zeit fallen auch die (geringmächtigen) kalkigen Ablagerungen des Mt. Brown (nördlich Christchurch), ebenso die fossile Cocos-Nuß von Coopers Beach.

Mittel- und Ober-Miozän: Korallen, Seeigel, Mollusken usw. des mitteltertiären thermischen Optimums verschwinden; es wird kühler (doch bleibt es wärmer als jetzt). Die jüngste fossile Cocos-Nuß, die ältesten Moa-Funde.

Pli o zän: Die miozänen Warmwasser-Formen verschwinden weiter, doch sind die Temperaturen nirgends kühler als an den heutigen Küsten der Nordinsel; auch die Pflanzen deuten auf etwas wärmeres Klima als heute (Nothofagus brassi-Pollen bis Dunedin).

Als besondere $\mathrm{Kennzeichen} \mathrm{des} \mathrm{neuseeländischen} \mathrm{Tertiärs} \mathrm{muß} \mathrm{man} \mathrm{her-}$ vorheben: keine Riffkalke und sonstige Zeugen tropischen Klimas, keine Salze und ähnliche Zeugen ariden Klimas. Auch rote Böden spielen keine besondere Rolle. D as Klima war gemäßigt bis subtropisch und humid. Es war offenbar etwas kühler als in Australien.

Die Temperatur-Kurve für die einzelnen Tertiär-Stufen ergibt nach dem, was wir oben feststellten (vgl. auch Abb. 9 nach D. F. SQuires) e in Maximum im mittleren Tertiär, ganz ähnlich wie in Australien (wo sie außerdem durch 018/016-Bestimmungen belegt ist). Sie steht aber im Gegensatz zu dem, was für Europa und Nordamerika bekannt ist. Dort ist das Alt-Tertiär wärmer als das Miozän. 


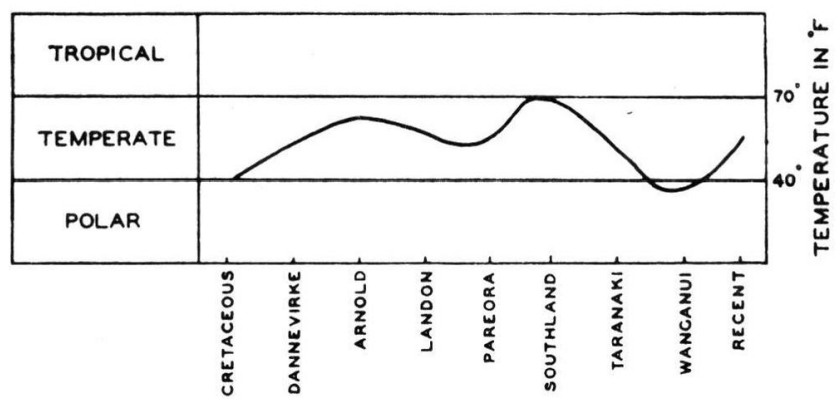

Abb. 9. Temperatur-Kurve des neuseeländischen Tertiärs. Nach D. F. SQuiREs aus E. GILL 1961. Dannevirke $=$ unt. Tertiär, Wanganui $=$ Pliozän + Alt-Quartär.

Das scheint im Widerspruch zu der Annahme zu stehen, daß das Tertiär auf der ganzen Erde einen ähnlichen Klimagang mit \pm konstanter Temperatur-Abnahme zeigt. Man kann diesen verschiedenen Verlauf der Kurven aber erklären, wenn man sie auffaßt als die Resultierende von zwei anderen Kurven (Abb. 10). Die eine Komponente wäre die Temperaturkurve, die durch die lokale B reitenlage und deren Änderungen gegeben ist. (Sollte die Breitenlage während des ganzen Tertiärs gleich geblieben sein, so wäre die entsprechende Temperaturkurve als eine Horizontale zu zeichnen.) Die zweite Komponente ergibt sich aus der a 11 gem e in en Tendenz des Klimas im Tertiär, allmählich kühler zu werden. Der Einfachheit halber ist ein geradliniger Kurvenverlauf
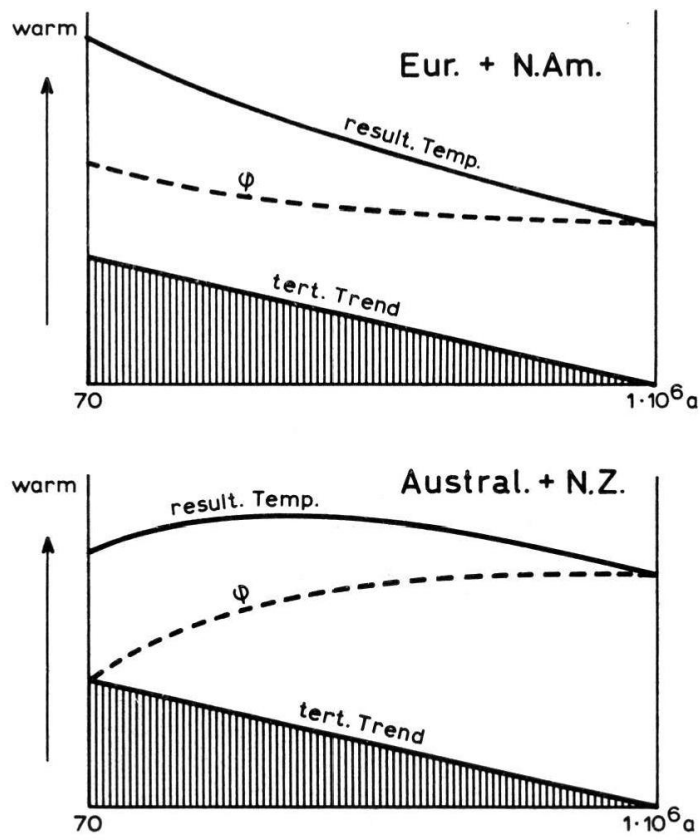

Abb. 10. Die tertiären Klima-Kurven als Resultierende zweier anderer Temperatur-Kurven. Tert. Trend $=$ allgemeine Tendenz im Tertiär $\phi=$ geogr. Breite und das davon abhängige Klima; result. Temp. $=$ resultierende Temperatur-Kurve, durch Addition der beiden anderen Kurven gewonnen. Oben: Europa-Nordamerika, unten: Australien und Neuseeland. 
angenommen. Wenn man voraussetzt, daß Australien auch im Tertiär (oder wenigstens im Alttertiär) die Tendenz der vorhergehenden Epochen fortsetzt, aus höheren in niedere Breiten zu wandern (vgl. die schematische Darstellung in Schwarzbach, 1965, Abb. 17, S. 154), dagegen Europa und Nordamerika genau die umgekehrte Tendenz oder aber (wofür in Europa mehr spricht) stabile Breiten-Lage aufweisen, so resultieren Temperatur-Kurven, die den tatsächlichen paläoklimatologischen Beobachtungen entsprechen.

\section{Prä-Tertiär}

Die prätertiäre Klimageschichte Neuseelands läßt sich - im Gegensatz zu Australien - nur ganz lückenhaft rekonstruieren. Für das Mesozoikum gilt allgemein dasselbe wie für das Tertiär. Es fehlen sowohl Kalke wie salinare Sedimente und fast gänzlich auch rote Sedimente. Allerdings darf man nicht vergessen, daß es sich meist um geosynklinale Ablagerungen handelt, welche die klimatischen Verhältnisse nur in beschränktem Umfange widerspiegeln. Immerhin ist der Gegensatz z. B. zu den Alpen frappierend. Eine Hauptrolle unter den Sedimenten spielen „Grauwacken“, d. h. nicht vollständig aufgearbeitete klastische Gesteine.

Im ganzen ist das mesozoische Klima also wohl - wie das tertiäre - n i c h t t ro pisch und nicht a rid gewesen. Das schließt nicht aus, daß es zeitweise etwas wärmer als heute war. In diesem Sinn hat man Funde von Cycadeen im älteren Mesosoikum ausgedeutet (vgl. P. Marshall 1912, S. 39); heute wachsen keine Cycadeen mehr an natürlichen Standorten in Neuseeland.

Das $\mathrm{Paläozoikum}$ ist nicht allzu vollständig. Bemerkenswert sind gelegentliche Kalk-Vorkommen im P e r m , so an der Basis der Matai-Serie, und sogar "riffbildende“ Korallen (im Ober-Perm). Von den permo-karbonischen Vereisungen ist nichts zu spüren. Das erinnert etwas an das Verhältnis des permischen Timor zu Australien, und man wird vielleicht erwägen müssen, ob nicht Neuseeland im Perm nördlicher als Australien lag.

\section{R ü ckblick}

Für A ustralien ergibt sich - wie ich früher auseinandergesetzt habe - das klare Bild einer W a n der ung aus kaltem Klima in subtropisch-tropische Bereiche im Laufe der letzten 250-300 Mill. Jahre (vgl. 1965, Abb. 17). Die paläomagnetischen Messungen haben zu einem gleichen Ergebnis geführt.

Das Klima Neuse ela nds dagegen hat sich anscheinend we niger deutlich ge w a n delt. Mesozoisches, tertiäres und heutiges Klima sind offenbar nicht allzu sehr voneinander unterschieden; die Schwankungen, die man z. B. im Tertiär beobachtet, sind von relativ geringer Größenordnung. Zum mindesten ist - anders als in Australien eine einheitliche Gesamt-Trendenz nicht zu erkennen. Ja, die (freilich spärlichen) Klimazeugen aus dem Perm deuten vielleicht sogar auf kurzfristig umgekehrte Tendenz.

Wenn diese Unterschiede wirklich reell und nicht durch lückenhafte Überlieferung (besonders in Neuseeland) vorgetäuscht sind, könnte das dadurch bedingt sein, daß beide Gebiete unabhängig voneinander wanderten (Australien schnell, Neuseeland langsamer).

Dank. Auf meinen Reisen konnte ich mich der sachkundigen und uneigennützigen Hilfe neuseeländischer Kollegen erfreuen, ohne die ich das große Programm nicht hätte durchführen können. Ich habe besonders zu danken: R. S. Allan (Christchurch), M. GAGE (Christchurch), D. R. GregG (Christchurch), W. F. Harris (Lower Hutt), J. Healeey (Rotorua), G. J. Lensen (Lower Hutt) und J. D. RaEsIDE (Dunedin). Der Deutschen Forschungsgemeinschaft bin ich für eine Beihilfe, Dr. L. Ahorner (Bensberg) für kritische Durchsicht des Manuskripts zu großem Dank verpflichtet. 


\section{Schriftenverzeichnis}

Cockayne, L.: The vegetation of New Zealand. - 364 S., Leipzig - New York 1921.

Cotron, C. A.: Geomorphology. 7. Aufl., 505, S., Christchurch 1958.

Couper, R. A. \& McQuenn, D. R.: Pliocene and Pleistocene plant fossils of New Zealand and their climatic interpretation. - New Zeald. J. Sci. Techn., B, 35, 398-420, Wellington 1954.

Cranwell, L. M. \& v. Post, L.: Post-Pleistocene pollen diagrams from the southern Hemisphere. Geogr. Ann. 18, 308-347, Stockholm 1936.

Eiby, G. A.: Earthquakes. - 168 S., London 1957.

Fleming, C. A.: The geology of Wanganui subdivision. - - N. Z. Geol. Surv., Bull. 52, 1-362, Wellington 1953. - - New Zealand biogeography. - Tuatara, 10, 53-108, 1962. - Palaeontology and southern biogeography. - In: J. L. Gressitr (ed.), Pacific basin biogeography, 369-385, Honolulu 1963.

Gage, M. \& Suggate, R. P.: Glacial chronology of the New Zealand Pleistocene. - Geol. Soc. Am. Bull. 69, 589-598, N.York 1958.

GILL, E. D.: The climates of Gondwanaland in Kainozoic times. - In: A. E. M. NaIrN (ed.), Descriptive palaeoclimatology, 332-35,3, N.York 1961.

Grindley, G. W., Harrington, H. J. \& Wood, B. L.: The geological map of New Zealand. 111 S., Wellington 1961.

GunN, B. M.: Flow rates and secondary structures of Fox and Franz Josef Glaciers, New Zealand. J. Glac. 5, 38, 173-190, Cambridge 1964.

Kolb, A.: Historische Gletscherschwankungen auf der Südhalbkugel, insbesondere auf Neuseeland. Geogr. Forschungen, Festschr. Hans KINZL, 123-146, Innsbruck 1958.

Lensen, G. J.: The Wellington fault from Cook Strait to Manawatu gorge. - N.Z.J. Geol. Geophys. 1, 178-196, Wellington 1958.

Marshall, P.: New Zealand and adjacent islands. - Hdb. region. Geol., VII, 1, 1-77, Heidelberg 1912.

Mercer, J. H.: Glacier variations in New Zealand. - IGY World Data Center A, Glaciol., Amer. Geogr. Soc., 33-44. N.York 1962.

Raeside, J. D.: Loess deposits of the South Island, New Zealand, and soils formed on them. N.Z. J. Geol. Geophys., 7, 811-838, Wellington 1964.

Schwarzbach, M.: Edaphisch bedingte Wüsten. - Z. Geomorph. 8, 440-452, Berlin 1964. - Paläoklimatologische Eindrücke aus Australien. - Geol. Rdsch. 54, 1, 128-160, Stuttg. 1965.

Walter, H. \& Lieth, H.: Klimadiagramm-Weltatlas. Jena 1960.

Wellmann, H. W.: New Zealand Quaternary tectonics. - Geol. Rdsch. 43, 248-257, Stuttgart 1955.

Willett, R. W.: The New Zealand Pleistocene snow line. - N.Z.J. Sci. Techn., B 32, 18-48, Wellington 195,0 .

Woldstedt, P.: Die Vergletscherungen Neuseelands und die Frage der Gleichzeitigkeit mit den europäischen Vereisungen. - Eiszeitalter und Gegenwart 12, 5-8. Öhringen 1962. - - Das Eiszeitalter. III. - 328 S., Stuttgart 1965.

Manuskr. eingeg. 1. 7. 1965.

Anschrift des Verf.: Prof. Dr. M. Schwarzbach, 5 Köln, Zülpicher Str. 49, Geol. Institut der Univ. 